\title{
Surgical procedures for treatment of adult acquired flatfoot deformity: a network meta-analysis
}

\author{
Xu Tao, Wan Chen and Kanglai Tang*
}

\begin{abstract}
Background: Adult acquired flatfoot deformity (AAFD) represents a spectrum of deformities affecting the foot and the ankle. The optimal management of AAFD remains controversial. We evaluated the efficacy of surgical treatments of AAFD using both direct and indirect evidences.

Methods: We searched PubMed, EmBase, and the Cochrane Library to identify eligible studies conducted through November 2018. To compare different surgical strategies, we performed a network meta-analysis. A traditional meta-analysis using a random-effects model was used to evaluate the pooled outcome.

Results: A total of 21 studies including 498 patients were collected and analyzed. Network meta-analysis results based on lateral angle talocalcaneal-calcaneal pitch (LAT-CP) indicated that medial displacement calcaneal osteotomy (MDCO) has the highest probability to be the best course of AAFD treatment. However, analyses based on anteroposterior talo-first metatarsal (AP-TMT1) and lateral angle talocalcaneal talo-first metatarsal (LAT-TMT1) suggested that lateral column lengthening $(\mathrm{LCL})$ was the best treatment, while those based on lateral angle talocalcaneal-arch height, anteroposterior talocalcaneal (AP-TC), lateral angle talocalcaneal-talocalcaneal (LAT-TC), anteroposterior-talonavicular coverage (AP-TNC), talonavicular coverage (TNC), and the American Orthopedic Foot and Ankle Society (AOFAS) indicated triple arthrodesis (TAO) as the best treatment. Moreover, double arthrodesis (DAO) provided the best treatment effect on the function score. Furthermore, according to traditional meta-analysis, the summary of standardized mean differences (SMD) indicated that the surgical interventions are associated with significant improvements in LAT-CP (SMD - 1.78), LAT-arch height (SMD - 4.95), AOFAS (SMD - 5.24), AP-TMT1 (SMD 2.45), LAT-TMT1 (SMD 1.97), AP-TC (SMD 3.05), LAT-TC (SMD 2.20), AP-TNC (SMD 2.07), TNC (SMD 1.70), and function score (SMD 0.95).
\end{abstract}

Conclusions: Our findings indicated that MDCO, LCL, TAO, or DAO might be the best surgical approaches for AAFD treatment. Furthermore, patients who received surgical interventions had significant improvements in symptoms and function.

Keywords: Adult acquired flatfoot deformity, Surgical treatment, Meta-analysis

\footnotetext{
* Correspondence: tangkanglai@hotmail.com

Department of Orthopedic Surgery, Southwest Hospital, Army Medical

University, Chongqing 400038, China
}

(c) The Author(s). 2019 Open Access This article is distributed under the terms of the Creative Commons Attribution 4.0 International License (http://creativecommons.org/licenses/by/4.0/), which permits unrestricted use, distribution, and reproduction in any medium, provided you give appropriate credit to the original author(s) and the source, provide a link to the Creative Commons license, and indicate if changes were made. The Creative Commons Public Domain Dedication waiver (http://creativecommons.org/publicdomain/zero/1.0/) applies to the data made available in this article, unless otherwise stated. 


\section{Background}

Adult acquired flatfoot deformity (AAFD) is a degenerative disease characterized by pathological changes in the tibialis posterior tendon, spring ligament complex, deltoid ligament, and other ligaments of the hindfoot [1]. This is divided into four stages. Stage I is characterized by pain and swelling in the tibialis posterior tendon but have normal alignment. Stage II is characterized by flexible deformities in the foot and can also include the hindfoot valgus, varying arch height, and degrees of abduction and supination of the forefoot. Stage III is similar to stage II, but the deformities are more severe and are rigid rather than flexible. Lastly, stage IV is characterized by fixed deformities and valgus tilting of the talus [2-4]. The pathogenesis of AAFD still remains unclear. The possible pathogenic factors include varying stresses on surrounding joints and weakening of the dynamic and static ligamentous restraints of the hindfoot and midfoot [1].

The treatment of AAFD involves slowing down the progression of the disease. Surgical techniques, such as soft tissue with medial or lateral column procedures, are widely used to avoid the progression of these fixed deformities [1]. According to a previous study, the medial surgical approach permitted fusion without the development of non-union and provided a significant correction of the fixed deformities. However, there are limited studies that compared the efficacy among these surgical techniques, and the best approach for treating AAFD still remains controversial.

Currently, the common surgical procedures included medial displacement calcaneal osteotomy (MDCO), lateral column lengthening (LCL), modified triple arthrodesis (MTA), and flexor digitorum longus (FDL) transfer. These strategies are widely used for mild deformities with flexibility, whereas no quantitative indexes are constructed for operative indication. Previous studies have showed that these interventions can slow down the progression and relieve the clinical symptoms of AAFD, but a clear comparison of the efficacy among these surgical procedures is still needed $[5,6]$. Hence, this network meta-analysis aimed to use both direct and indirect evidences to evaluate the comparative efficacy of surgical procedures in AAFD patients.

\section{Methods}

\section{Data sources, search strategy, and selection criteria}

This review was conducted and reported according to the Preferred Reporting Items for Systematic Reviews and Meta-Analysis Statement, 2009 (Additional file 1: PRISMA Checklist) [7].

We performed systematic searches in PubMed, EmBase, and the Cochrane Library for relevant literature that is published on or before November 2018. The following terms were used separately in the search: "posterior tibial tendon dysfunction," "pes planus," "adult acquired flatfoot deformity," "midfoot abduction," "posterior tibial tendon," "pes planovalgus," "posterior tibial tendon insufficiency," "flatfoot staging," "flatfoot treatment," "foot orthoses," and "surgical". The resulting titles and abstracts of the primary collections were browsed. To identify additional candidate studies, the reference lists of the included studies and reviews were also evaluated.

The literature search was performed by two independent authors, and any inconsistencies between them were settled by group discussion until a consensus was reached. A study was considered eligible for inclusion if the following criteria were met: (1) the trials that investigated surgical procedures for the treatment of AAFD patients and (2) the authors' outcome reports included lateral angle talocalcaneal-calcaneal pitch (LAT-CP), anteroposterior talo-first metatarsal (AP-TMT1), lateral angle talocalcaneal talo-first metatarsal (LAT-TMT1), LAT-arch height, anteroposterior talocalcaneal (AP-TC), lateral angle talocalcaneal-talocalcaneal (LAT-TC), anteroposterior-talonavicular coverage (AP-TNC), TNC, the American Orthopedic Foot and Ankle Society (AOFAS), and function score. Cohorts, case controls, case series, reviews, and editorials were excluded due to uncontrolled confounders.

\section{Data collection and quality assessment}

Two reviewers independently extracted all the data, and disagreements were resolved in consultation with third-party investigators. The following information was extracted from the included articles: first author, publication year, country, prospective or retrospective design, age, follow-up duration, disease type, surgery type, reported outcomes, and number of patients. The investigated outcomes from radiography examination included LAT-CP, AP-TMT1, LAT-TMT1, LAT-arch height, AP-TC, LAT-TC, AP-TNC, TNC, AOFAS, and function score. Two reviewers independently assessed the quality of the included studies according to the Jadad scale in the following five domains: randomization ( 1 or 0$)$, concealment of the treatment allocation ( 1 or 0$)$, blinding ( 1 or 0 ), completeness of follow-up ( 1 or 0 ), and the use of intention-to-treat analysis ( 1 or 0 ). Based on these results, the studies were given scores with a previously developed scoring system for quality assessment that ranged from 1 to 5 [8].

\section{Statistical analysis}

For traditional meta-analyses, we used the inverse variance method to pool the continuous data. The results are presented as standardized mean difference (SMD) with 95\% confidence intervals (95\%CIs). In our network meta-analysis, we used a random-effect network meta-analysis for mixed multiple treatment comparisons, 
which fully preserves the within-trial randomized treatment comparisons of each trial [9].

The $I^{2}$ statistic was calculated to evaluate the extent of variability that was attributable to statistical heterogeneity between the trials. In the absence of statistical heterogeneity $\left(I^{2}<50 \%\right)$, we used a fixed-effects model; otherwise, we used a random-effects model in a traditional meta-analysis $[10,11]$. Consistency within every closed triangle or quadratic loop was investigated using a loop-specific approach in our network meta-analysis. During analysis, inconsistency factors and their $95 \%$ CIs were used to determine their compatibility with zero [12]. To rank the treatments for an outcome, we used surface under the cumulative ranking (SUCRA) probabilities [13]. A "comparison-adjusted" funnel plot was used to assess the presence of small-study effects in our network meta-analysis [14]. All tests were two-tailed, and $p$ value of less than 0.05 was deemed to be statistically significant. We analyzed the data using STATA software (version 10.0).

\section{Results}

Our initial search produced 1798 unique results. Of these, we collected 21 trials that met our study criteria, which included 498 patients [15-35] (Fig. 1). After reviewing the full texts, the reasons for the exclusion of literature included other intervention interferences, other similar diseases, and lack of desired outcome assessment. The general characteristics of the included studies are presented in Table 1. Of the included studies, 10 were conducted in the US, 5 in Europe, 4 in China, 1 in Japan, and 1 in Egypt. Study quality was evaluated using the Jadad scale (Table 1). Overall, 8 studies had a score of 2,10 studies had a score of 1 , and the remaining 3 studies had a score of 0 .

Traditional meta-analysis was used to evaluate the effect of surgical intervention for AAFD, and a summary of the outcomes is listed in Table 2. Overall, we noted that surgical intervention was associated with lower levels of LAT-CP (SMD - 1.78; 95\%CI -2.57 to - 0.99; $p<0.001 ; I^{2}$ 92.4\%), LAT-arch height (SMD - 4.95; $95 \% \mathrm{CI}-5.69$ to $\left.-4.20 ; p<0.001 ; I^{2} 0.0 \%\right)$, and AOFAS (SMD $-5.24 ; 95 \%$ CI -6.98 to $-3.49 ; p<0.001 ; I^{2}$ 90.7\%). Furthermore, surgical treatments were associated with AAFD patients with higher levels of AP-TMT1 (SMD 2.45; 95\%CI 1.74 to $3.17 ; p<0.001 ; I^{2} 91.5 \%$ ), LAT-TMT1 (SMD 1.97; 95\%CI 1.18 to $2.77 ; p<0.001 ; I^{2}$ 95.4\%), AP-TC (SMD 3.05; 95\%CI 1.37 to $4.73 ; p<$ 0.001; $I^{2}$ 96.2\%), LAT-TC (SMD 2.20; 95\%CI 0.98 to 3.42; $p<0.001 ; I^{2} 94.8 \%$ ), AP-TNC (SMD 2.07; 95\%CI 1.04 to $3.09 ; p<0.001 ; I^{2} 92.1 \%$ ), TNC (SMD 1.70; $95 \%$ CI 1.00 to $\left.2.40 ; p<0.001 ; I^{2} 83.1 \%\right)$, and function score (SMD $0.95 ; 95 \%$ CI 0.24 to $1.67 ; p=0.009 ; I^{2}$ 90.7\%). Although substantial heterogeneity was detected for most of the outcomes, the conclusions were not affected by the sequential exclusion of any single study.

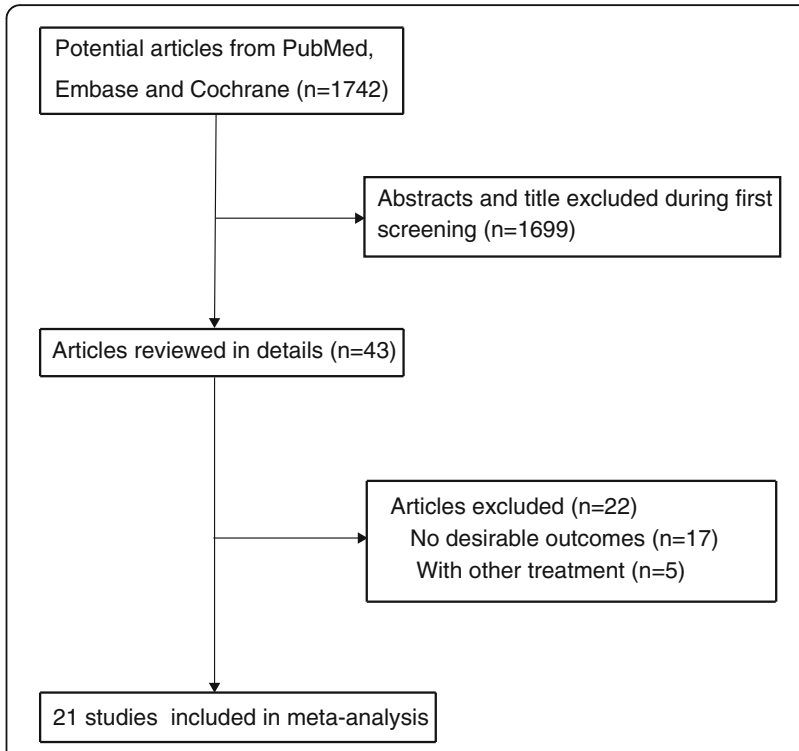

Fig. 1 Flow diagram showing the study selection process

The eligible LAT-CP comparisons in our network meta-analysis are predominantly pairwise comparisons of different surgical interventions for AAFD patients (Fig. 2). The modes in Fig. 2 are weighted according to the number of patients that received each surgical mode, and the edges are weighted according to the mean control group for all comparisons versus the corresponding preoperative values. The MDCO versus preoperative treatment demonstrated the highest contribution for the entire network meta-analysis. The LAT-CP results showed that the LAT-CP values following TAO (SMD 6.24; $95 \% \mathrm{CI}-11.59$ to -0.89 ) or MDCO (SMD - 9.31; $95 \% \mathrm{CI}-18.59$ to -0.12 ) are significantly lower than the corresponding preoperative values, but none of the other comparisons revealed significant differences.

A similar analysis suggested that LCL is the best treatment strategy based on the changes in AP-TMT1 (Fig. 3) and LAT-TMT1 values (Fig. 4). All types of surgical strategies for treating AAFD except for medial translational osteotomy (MTO) are associated with lower AP-TMT1 values (SMD - 2.60; 95\%CI - 9.01 to 3.80; Fig. 3). Additionally, the double arthrodesis (DAO), TAO, and LCL showed significant reduction in the levels of LAT-TMT1 as compared with corresponding preoperative values (Fig. 4). Finally, TAO showed the highest probability as the best treatment strategy based on changes in the LAT-arch height (Fig. 5), AP-TC (Fig. 6), ALT-TC (Fig. 7), AP-TNC (Fig. 8), TNC (Fig. 9), AOFAS (Fig. 10), and function scores (Fig. 11).

Both DAO and TAO were associated with higher LAT-arch height values (Fig. 5), increased AOFAS levels (SMD 33.48 and $95 \%$ CI 28.14 to 38.82 for DAO; SMD 44.30 and $95 \% \mathrm{CI} 32.58$ to 56.02 for TAO; Fig. 10), and 


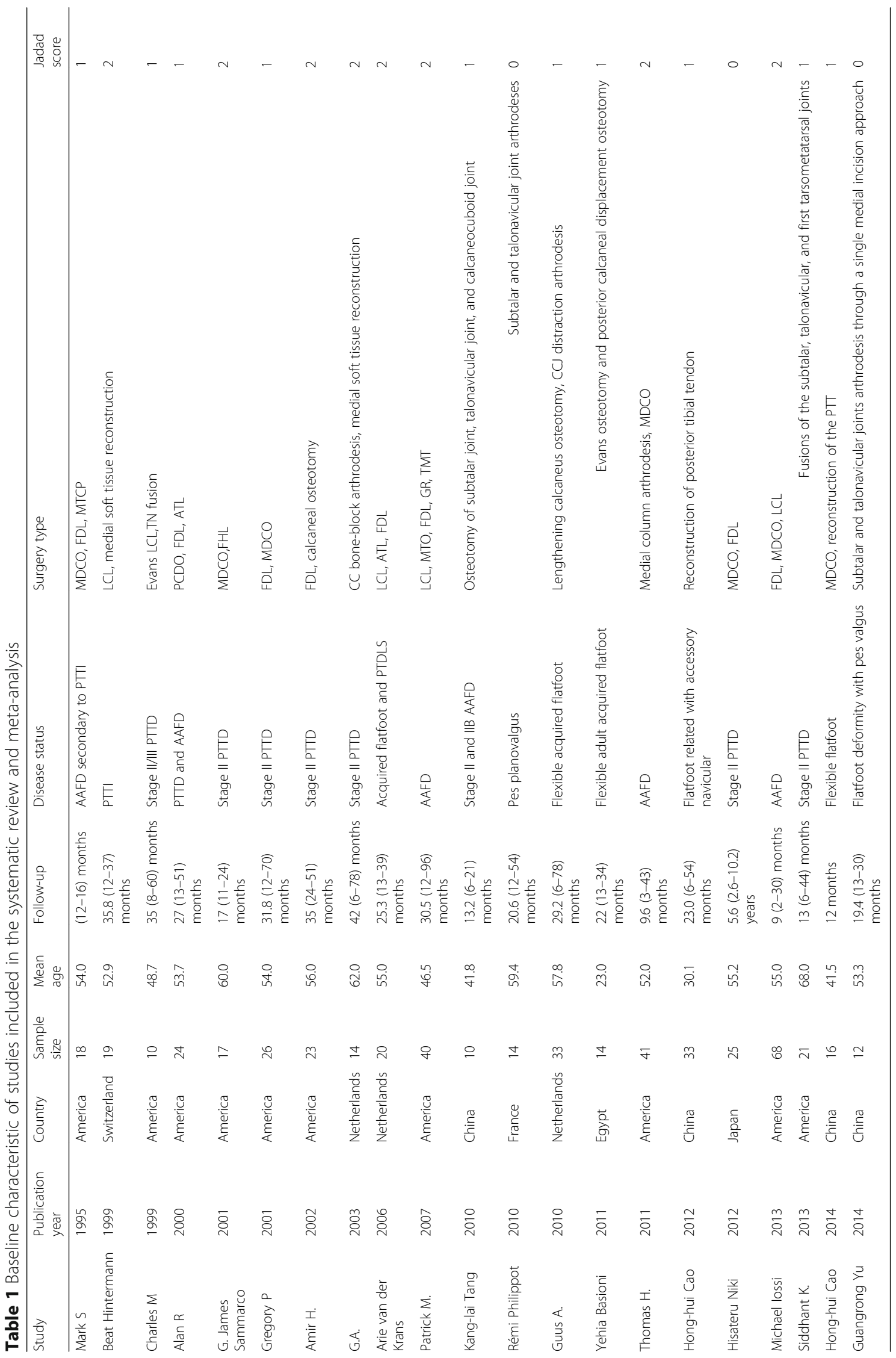


Table 2 Summary of the standard mean difference of all outcomes assessed by using traditional meta-analysis

\begin{tabular}{|c|c|c|c|c|c|}
\hline Outcomes & SMD & $95 \% \mathrm{Cl}$ & $p$ value & Heterogeneity (\%) & $p$ value for heterogeneity \\
\hline LAT-CP & -1.78 & -2.57 to -0.99 & $<0.001$ & 92.4 & $<0.001$ \\
\hline AP-TMT1 & 2.45 & 1.74 to 3.17 & $<0.001$ & 91.5 & $<0.001$ \\
\hline LAT-TMT1 & 1.97 & 1.18 to 2.77 & $<0.001$ & 95.4 & $<0.001$ \\
\hline LAT-arch height & -4.95 & -5.69 to -4.20 & $<0.001$ & 0.0 & 0.398 \\
\hline AP-TC & 3.05 & 1.37 to 4.73 & $<0.001$ & 96.2 & $<0.001$ \\
\hline LAT-TC & 2.20 & 0.98 to 3.42 & $<0.001$ & 94.8 & $<0.001$ \\
\hline AP-TNC & 2.07 & 1.04 to 3.09 & $<0.001$ & 92.1 & $<0.001$ \\
\hline TNC & 1.70 & 1.00 to 2.40 & $<0.001$ & 83.1 & $<0.001$ \\
\hline AOFAS & -5.24 & -6.98 to -3.49 & $<0.001$ & 90.7 & $<0.001$ \\
\hline Function score & 0.95 & 0.24 to 1.67 & 0.009 & 90.7 & $<0.001$ \\
\hline
\end{tabular}

lower ALT-TC levels (SMD - 6.71 and 95\%CI - 12.54 to -0.89 for DAO; SMD -11.91 and $95 \% \mathrm{CI}-17.96$ to 5.87 for TAO; Fig. 7). Patients who received DAO had significantly lower AP-TC values (SMD - 9.09; 95\%CI 17.00 to -1.18; Fig. 6), and those who received TAO had significantly reduced levels of AP-TNC (SMD 25.17; 95\%CI - 38.63 to - 11.71; Fig. 8). DAO and CJDA were associated with significantly lower levels of TNC (SMD -13.26 and $95 \% \mathrm{CI}-23.59$ to -2.93 for DAO; SMD -20.60 and $95 \% \mathrm{CI}-41.14$ to -0.06 for CJDA; Fig. 9). Furthermore, TAO was associated with lower function scores, whereas DAO significantly increased the function scores.

\section{Discussion}

This meta-analysis included 21 studies that evaluated surgical interventions in adult patients with AAFD. Our findings suggested that surgical interventions showed a significant impact on LAT-CP, AP-TMT1, LAT-TMT1, LAT-arch height, AP-TC, LAT-TC, AP-TNC, TNC, AOFAS, and function score. Additionally, we conducted the first network meta-analysis to analyze the effects of different types of surgery for the treatment of AAFD. The results of these analyses indicated that MDCO, LCL, TAO, and DAO might be superior to other surgical approaches for treating AAFD.

The methodological evaluation of each included study was limited by randomization, blinding, allocation concealment, withdrawals and dropouts, and the use of intention-to-treat analysis. In this study, all the included studies were self-contrast trials, and no trial required randomization, blinding, or allocation concealment. Although most of the trials reported withdrawals, dropouts, and the use of intention-to-treat analysis, other biases also contributed to heterogeneity in each study. Ultimately, taking into consideration the unsatisfactory

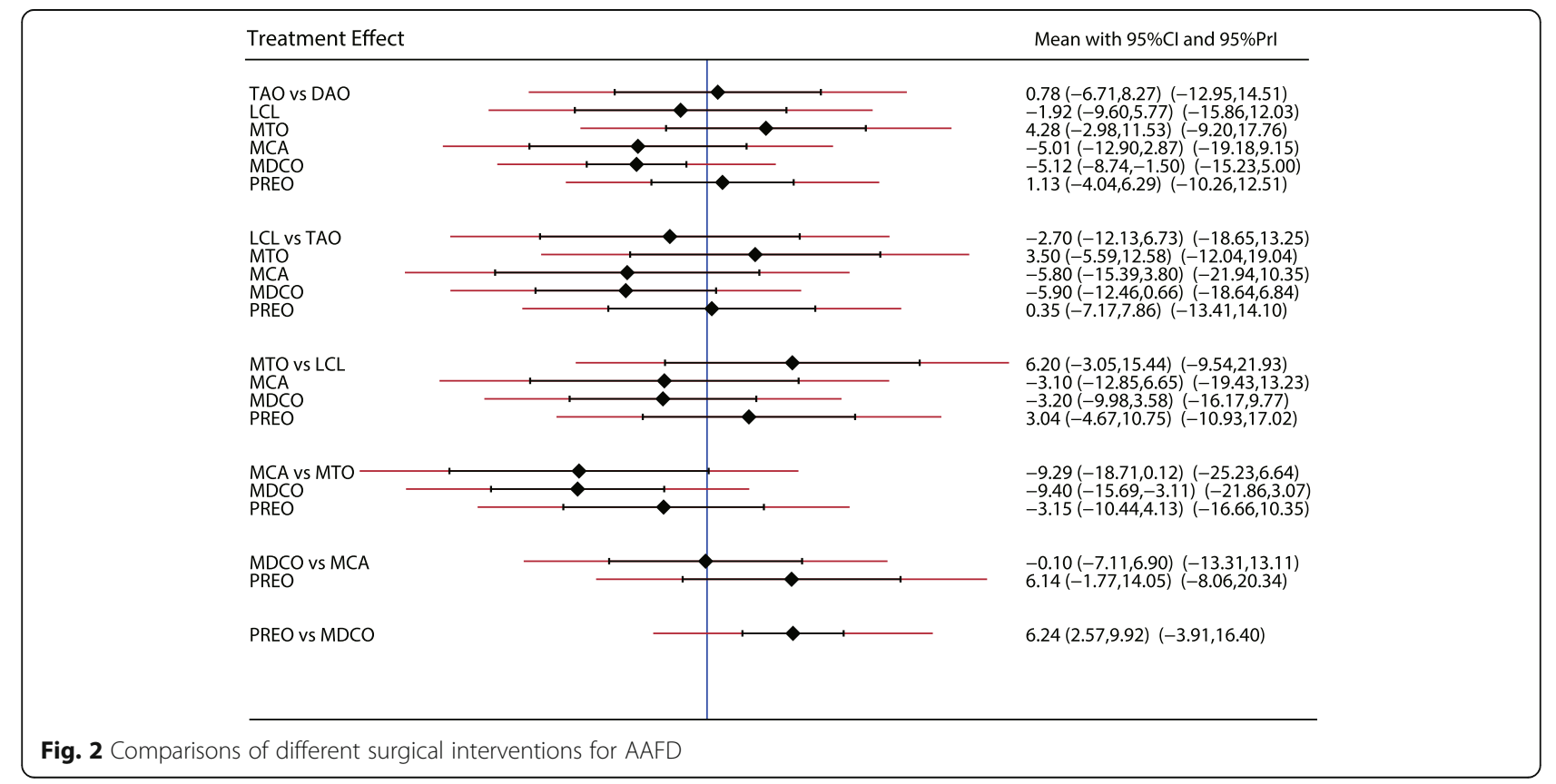




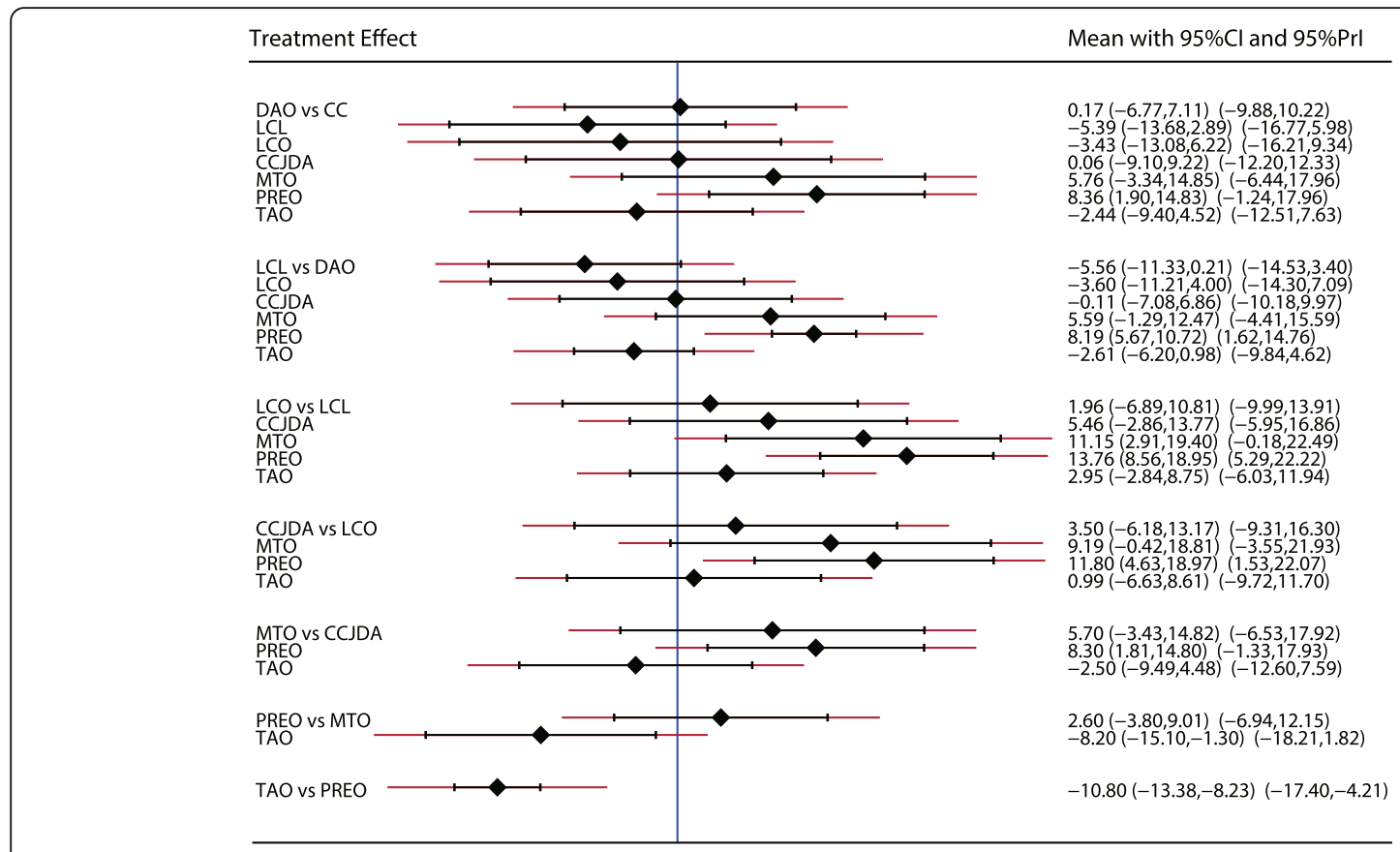

Fig. 3 The effect of different treatment strategies based on changes in AP-TMT1 values

quality of the included studies, we critically provided our recommendations for the treatment of patients with AAFD.

Cao et al. reported that MDCO with reconstruction of the posterior tibial tendon insertion on the navicular bone is considered as an effective treatment for flexible flatfoot with symptomatic accessory navicular and that this method is associated with excellent clinical outcomes and correction of the deformity [30]. Mehta et al. found that MTA showed a reliable and reproducible correction of the deformity seen in rigid stage III posterior tibial tendon dysfunction [33]. Iossi et al. suggested that clinical and radiographic parameters are also important to consider when choosing bony realignment procedures to reconstruct a flexible flatfoot deformity [32]. For the treatment of more severe deformities, LCL

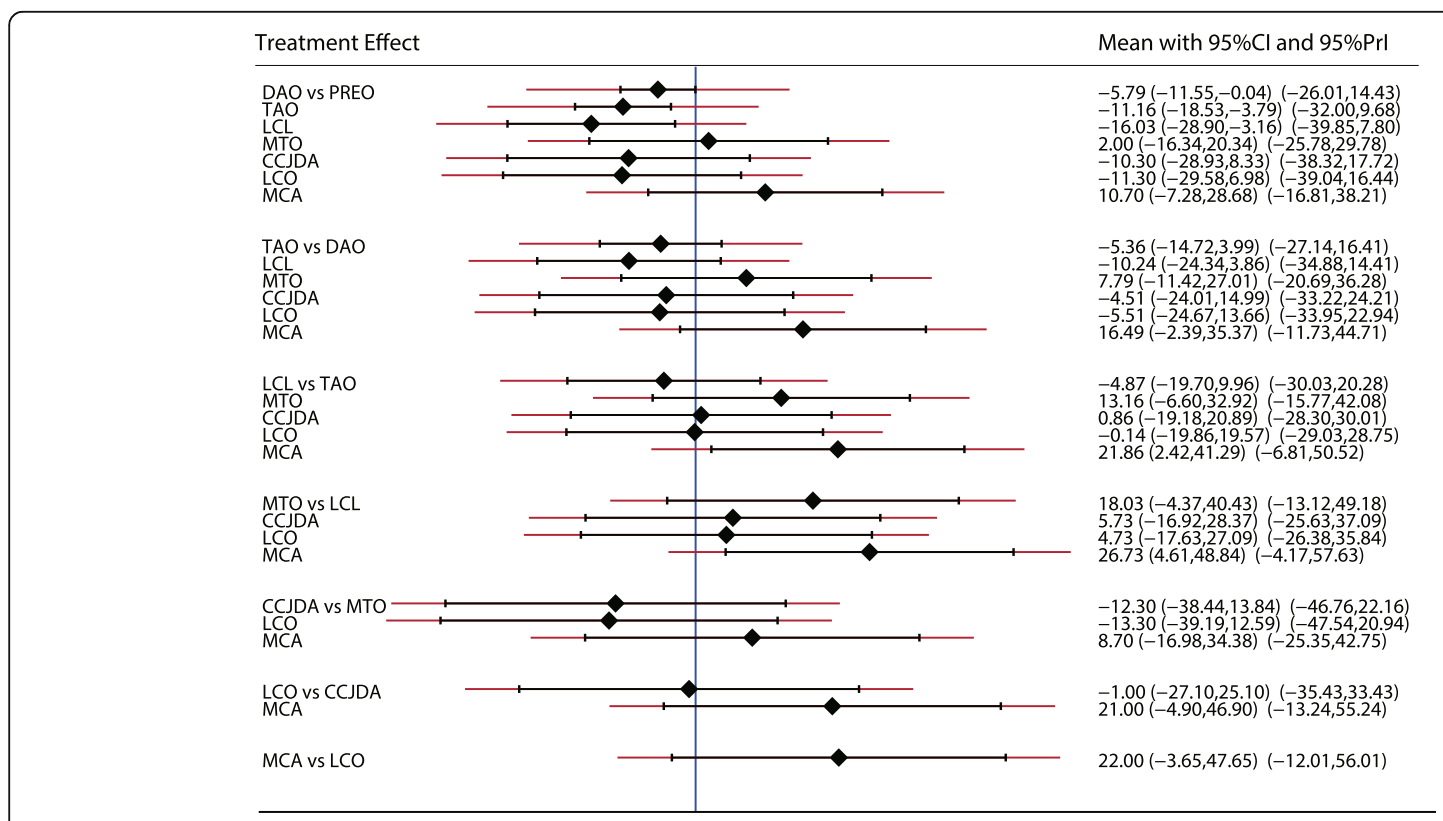

Fig. 4 The effect of different treatment strategies based on changes in LAT-TMT1 values 


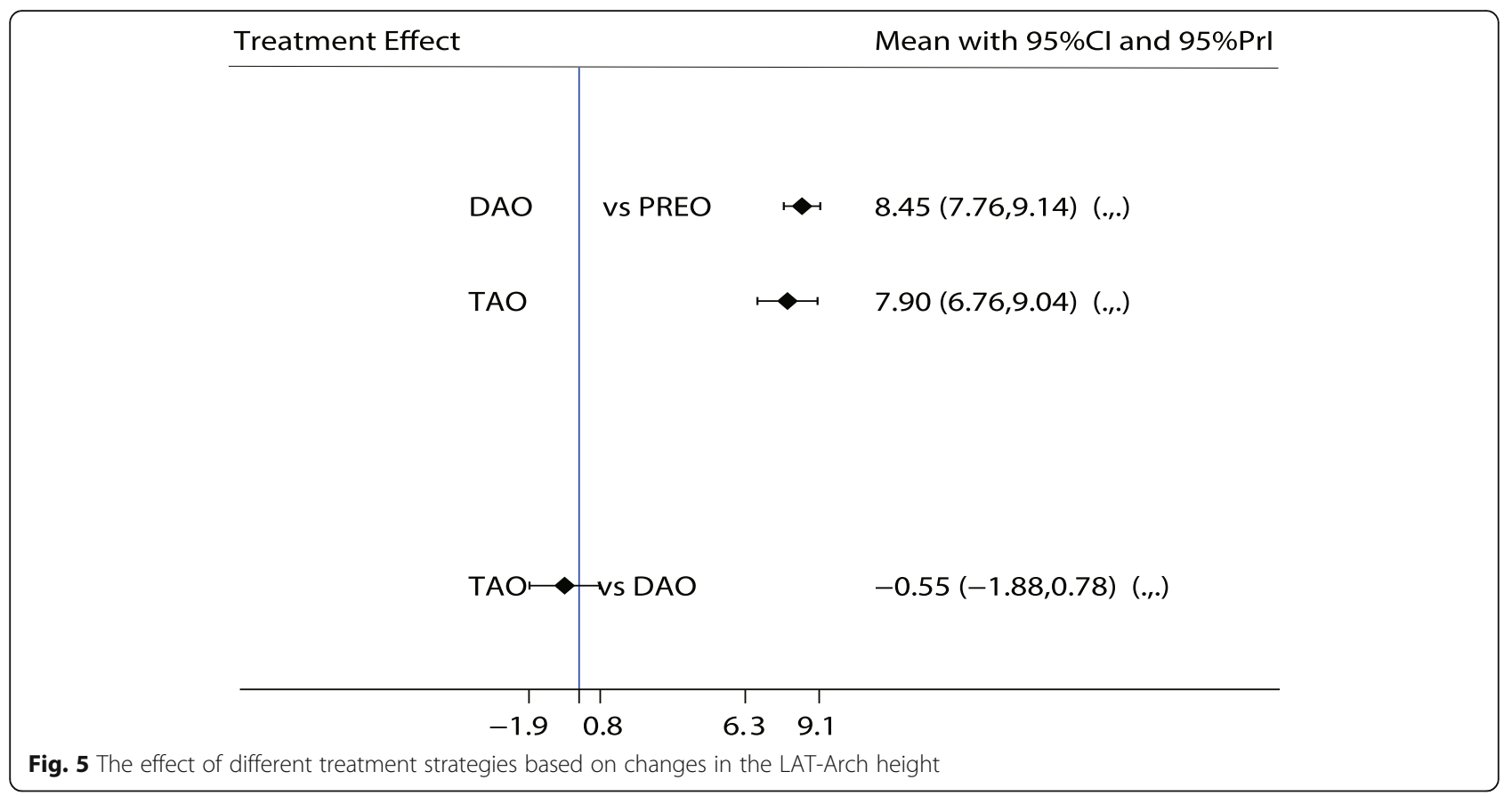

resulted in a greater radiographic improvement in alignment. A MDCO alone is also a valuable tool to correct these deformities, although it provides a different level of correction when compared with LCL. Overall, the diverse outcome assessments used in various interventions remains to be the greatest obstacle for the improvement of treatments for AADF, because it is difficult to compare these non-uniform results. Therefore, a systematic review was needed to define the comparison among the surgical approach treatments.

The intervention methods tested in our included studies were restricted to surgical treatments because they are widely used as the first-line treatments in AAFD patients. To increase the reliability of our

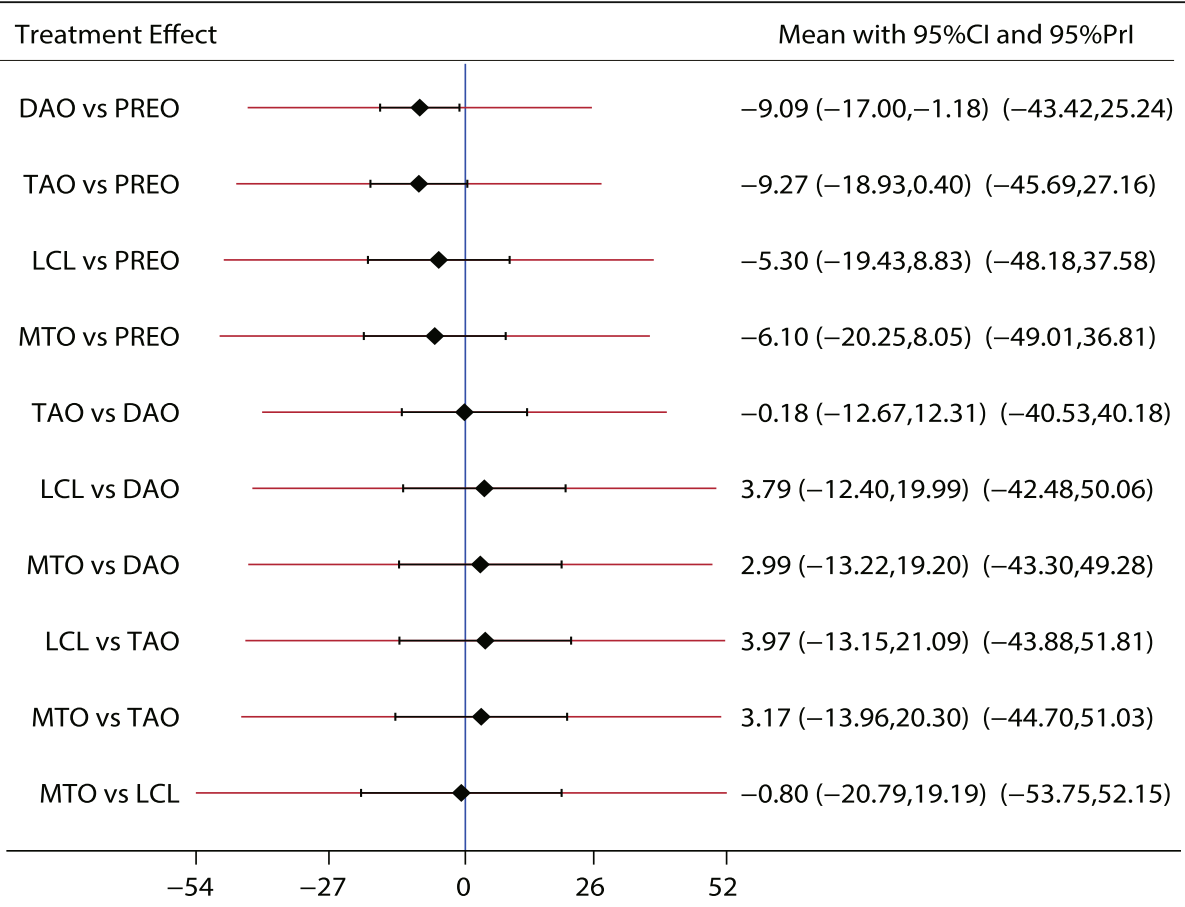

Fig. 6 The effect of different treatment strategies based on changes in AP-TC 


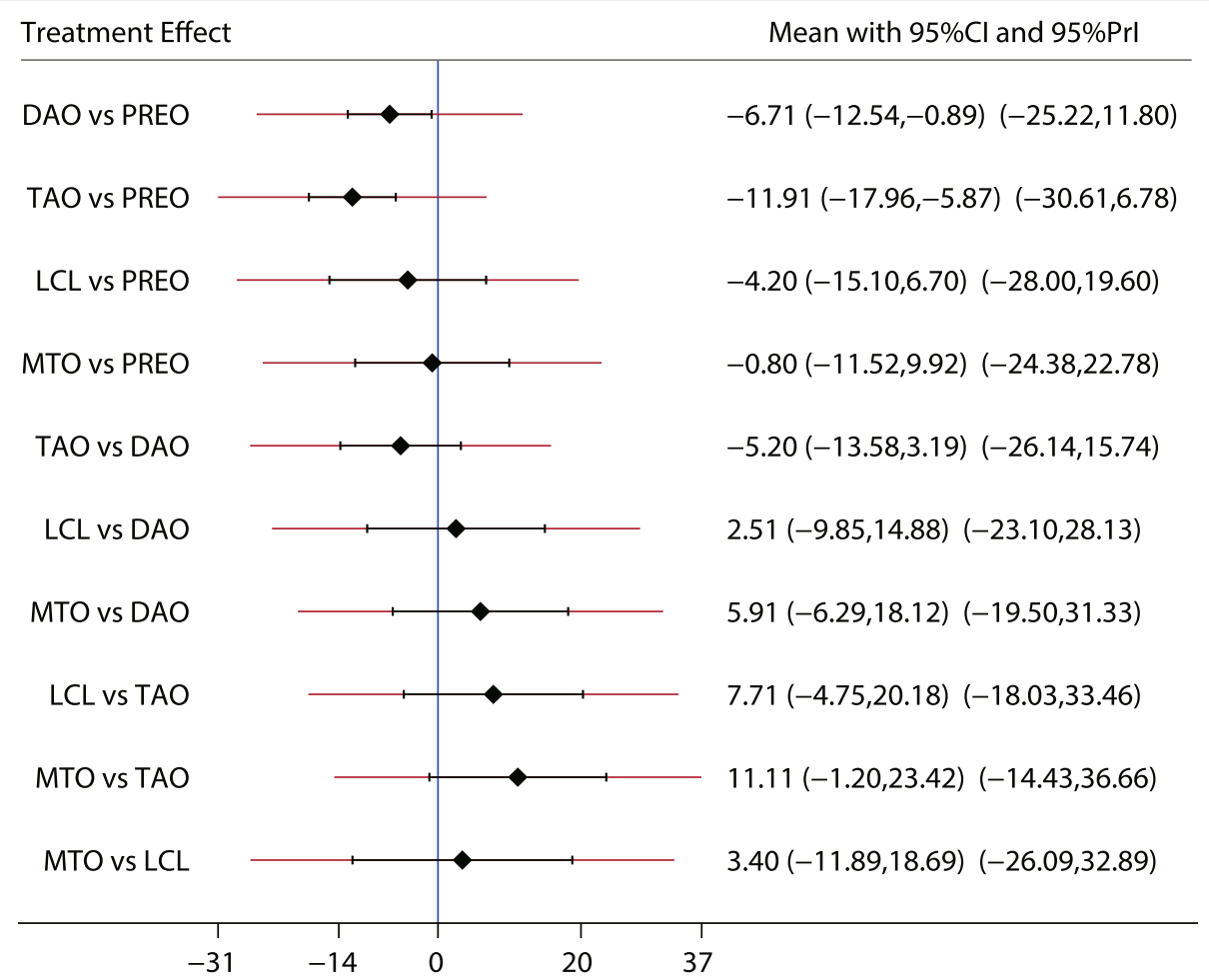

Fig. 7 The effect of different treatment strategies based on changes in ALT-TC

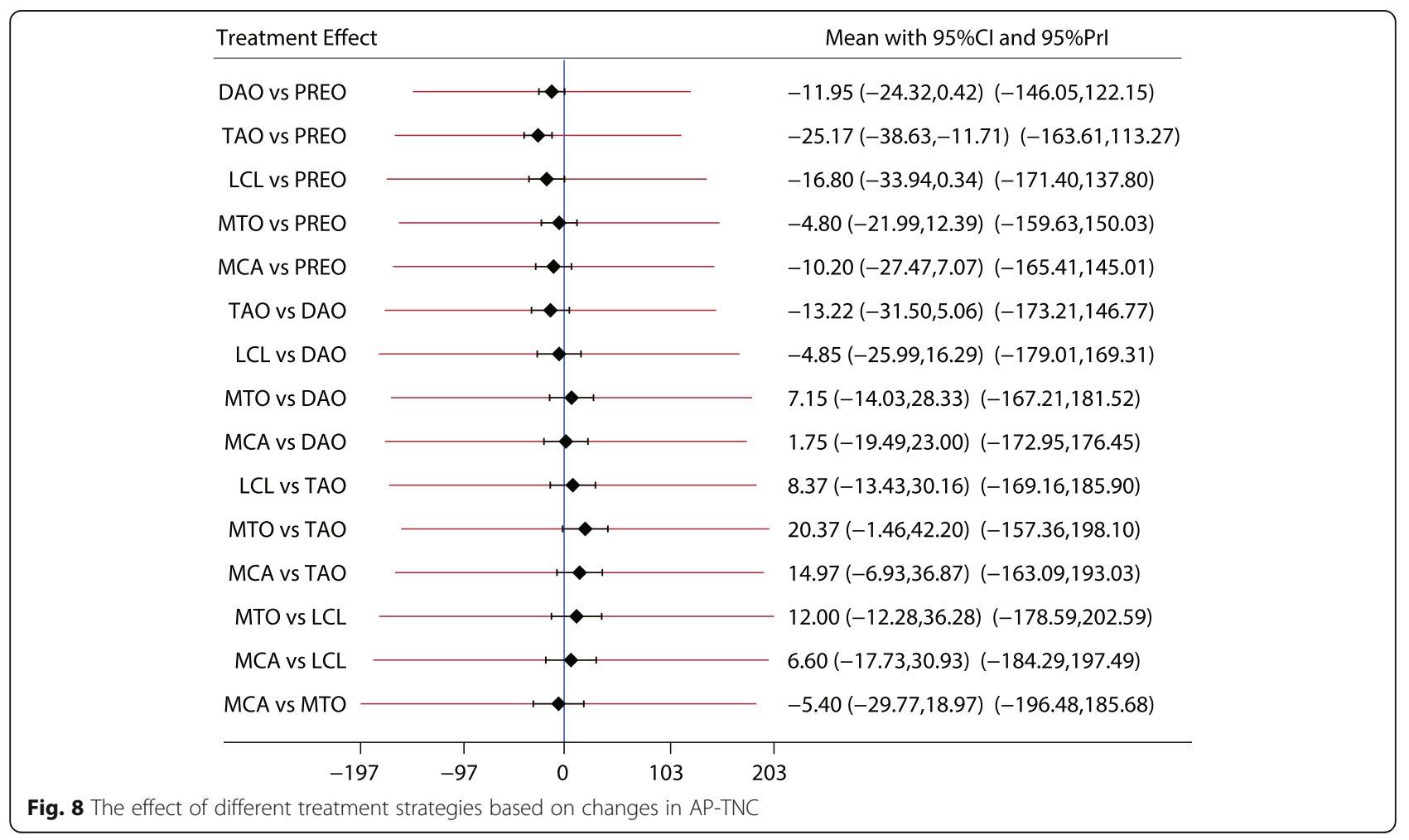




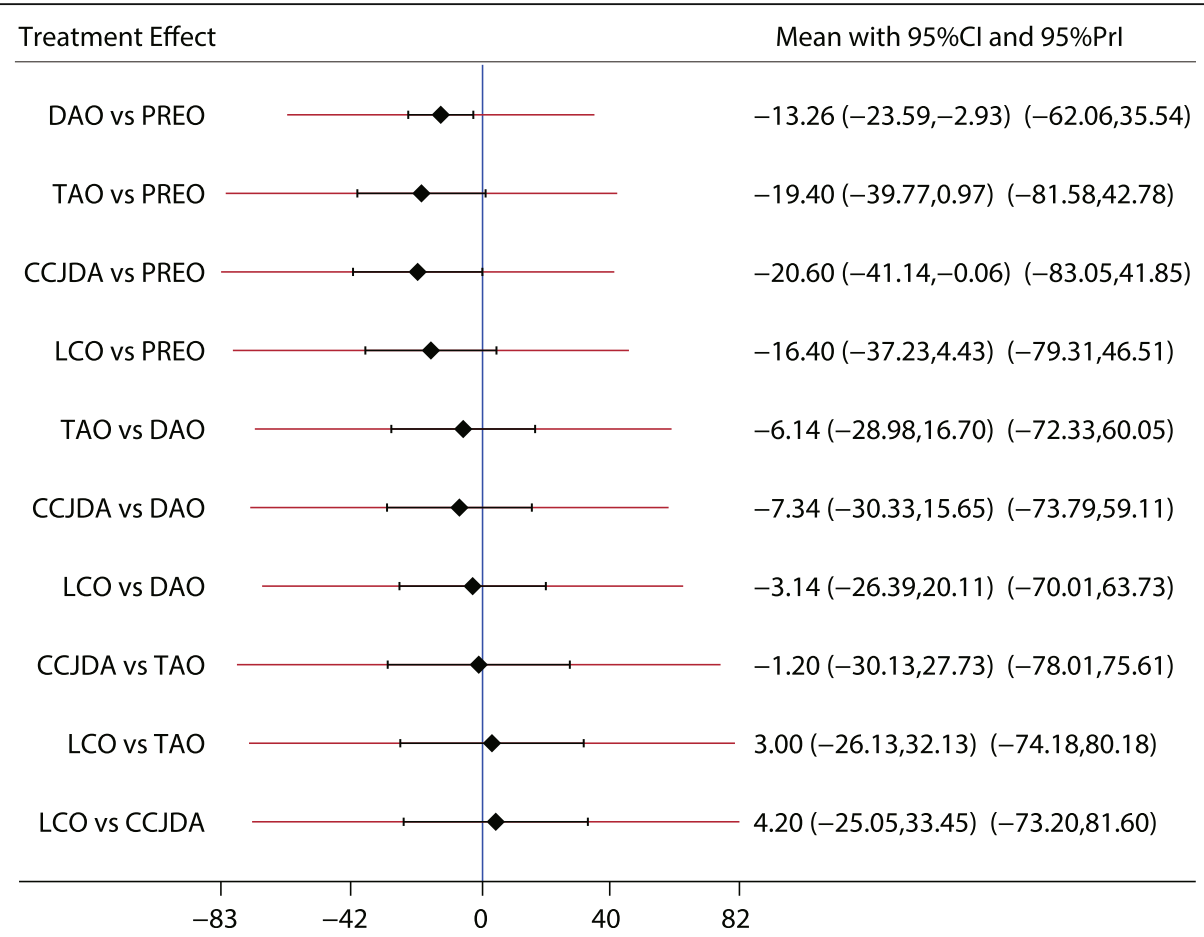

Fig. 9 The effect of different treatment strategies based on changes in TNC

study, we excluded the studies with high design bias because the treatment and outcome records of AADF, especially the pain and urgency records, are subjected to high subjectivity. We also comparatively analyzed the various surgical treatment approaches by network meta-analysis.
Various surgical types are currently used for AAFD treatment, but there is no widely accepted effective treatment to date. These surgical types include LCL, MTO, TAO, DAO, MCA, MCA, MDCO, excision of accessory navicular with reconstruction of posterior tibial tendon insertion on navicular, FDL, MTA, and reconstruction of the PTT. Our

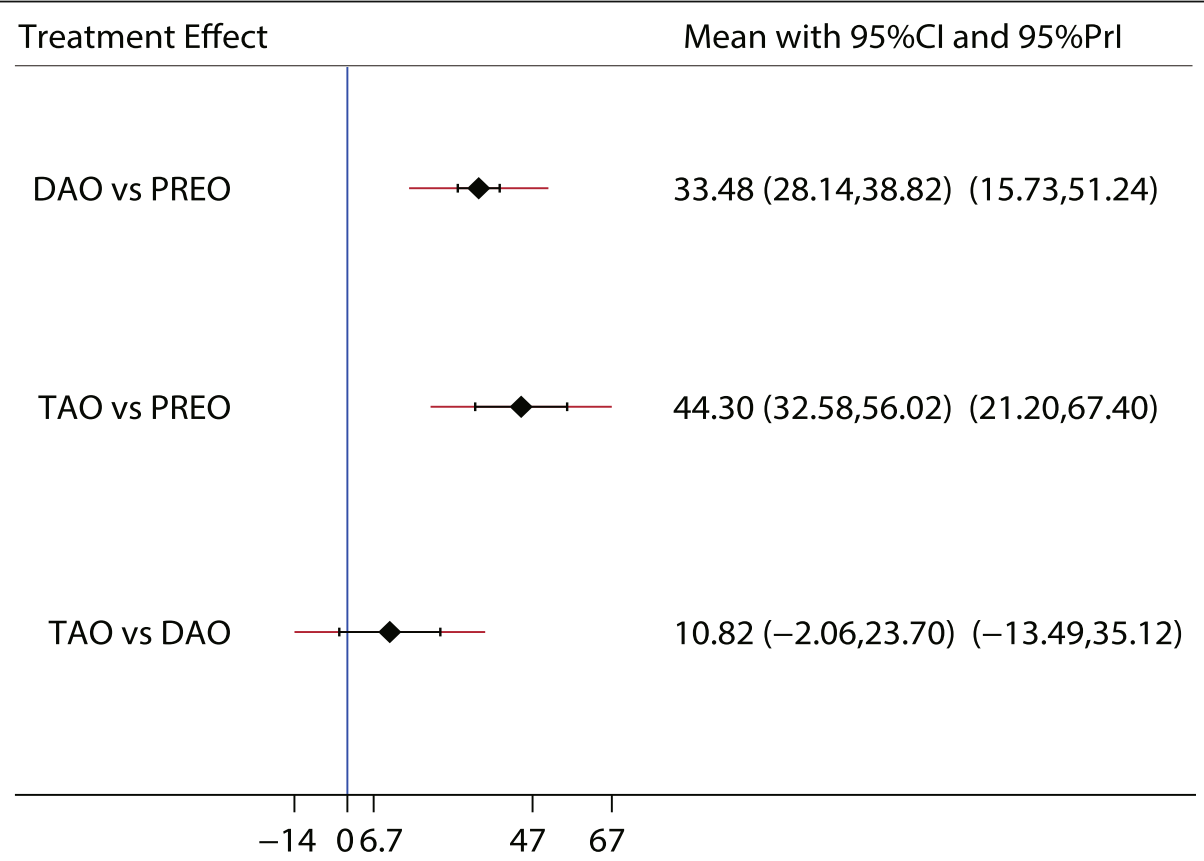

Fig. 10 The effect of different treatment strategies based on changes in AOFAS 


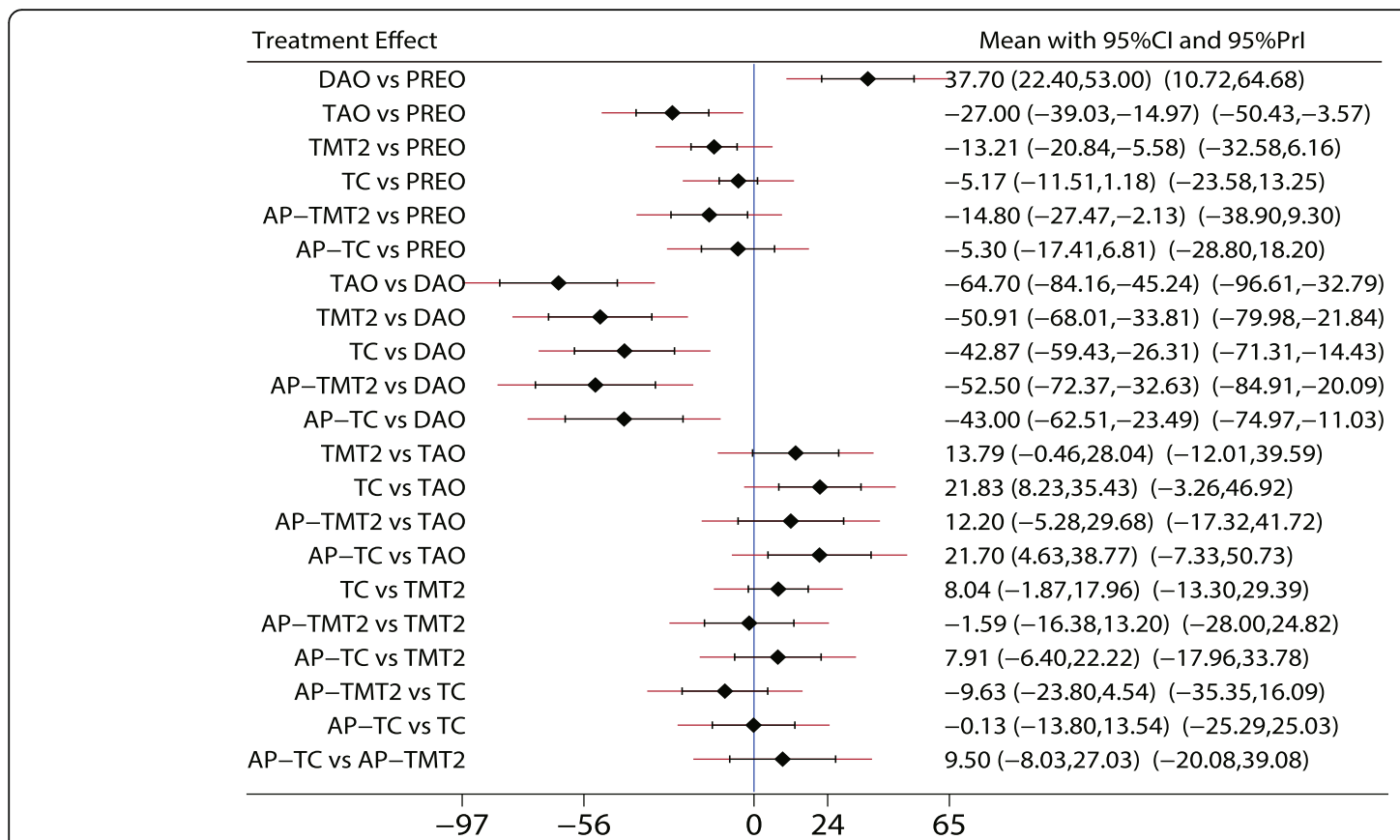

Fig. 11 The effect of different treatment strategies based on changes in the function scores

network meta-analysis suggested that among these methods, MDCO, LCL, and TAO might be considered as relatively better surgical strategies for the treatment of patients with AAFD.

The results of our traditional meta-analysis revealed that AAFD patients who received a surgical intervention showed significant improvement with LAT-CP, AP-TMT1, LAT-TMT1, LAT-arch height, AP-TC, LAT-TC, AP-TNC, TNC, AOFAS, and function score values when compared with their preoperative levels. However, several studies reported inconsistent results. Firstly, Iossi et al. showed that a FDL transfer to the navicular and bony realignment showed no significant impact on the function score, whereas the LCL results showed a greater radiographic improvement in alignment [32]. Furthermore, Haeseker et al. suggested that LCL by means of calcaneus osteotomy rather than distraction arthrodesis of the calcaneocuboid joint assists in the correction of stage II posterior tibial tendon dysfunction, but showed no effect on the function score [27]. As there were only few trials reported for each specific surgical strategy, and so we could not conduct a stratified analysis based on the types of surgical intervention. However, we were able to comprehensively compare these methods by performing a network meta-analysis.

Our study has few limitations. Firstly, we did not have access to specific data of individuals for all the trials, and so our statistical analysis could only be performed at a study level. Secondly, there are limited studies using single treatment, which in turn could reduce the reliability of our meta-analysis. Thirdly, there was heterogeneity in most of the outcomes among the included studies, attributing to the non-standardization of outcome assessment. Fourthly, the pre-operative stages of patients might affect the treatment effects of surgical strategies, whereas most of the included studies did not provide these information in detail. Finally, we were not able to use a subgroup analysis and meta-regression to reduce the heterogeneity because there were too few studies using a single surgical intervention for the treatment of AADF. Therefore, unifying the outcome standard is very important for further research to improvise the treatments for AADF.

\section{Conclusion}

The findings of this study suggested that surgical intervention in AADF patients is associated with beneficial prognosis. Furthermore, MDCO, LCL, and TAO are the three surgical strategies that showed the most effective treatments in patients with AAFD. Further research would not only benefit from the addition of well-designed studies, but also from the publication of studies that focused on the pathogenesis and therapeutic mechanisms of AADF, which further improves the understanding of the disease and its treatments. 


\section{Additional file}

Additional file 1: PRISMA Checklist. (DOC $72 \mathrm{~kb}$ )

\begin{abstract}
Abbreviations
AAFD: Adult acquired flatfoot deformity; AOFAS: American Orthopedic Foot and Ankle Society; AP-TC: Anteroposterior talocalcaneal; AP-

TMT1: Anteroposterior talo-first metatarsal; AP-TNC: Anteroposteriortalonavicular coverage; DAO: Double arthrodesis; FDL: Flexor digitorum longus; LAT-CP: Lateral angle talocalcaneal-calcaneal pitch; LAT-TC: Lateral angle talocalcaneal-talocalcaneal; LCL: Lateral column lengthening; MDCO: Medial displacement calcaneal osteotomy; MTA: Modified triple arthrodesis; SMD: Standardized mean differences; SUCRA: Surface under the cumulative ranking; TAO: Triple arthrodesis
\end{abstract}

\section{Acknowledgements \\ Not applicable.}

\section{Funding}

Not applicable.

\section{Availability of data and materials}

The data set supporting the results of this article are included within the article.

\section{Authors' contributions}

XT conceived and coordinated the study, designed, performed, and analyzed the experiments, and wrote the paper. WC carried out the data collection, data analysis, and revised the paper. KT designed the study, carried out the data analysis, and revised the paper. All authors reviewed the results and approved the final version of the manuscript.

\section{Ethics approval and consent to participate}

Not applicable.

\section{Consent for publication}

Not applicable.

\section{Competing interests}

The authors declare that they have no competing interests.

\section{Publisher's Note}

Springer Nature remains neutral with regard to jurisdictional claims in published maps and institutional affiliations.

Received: 29 September 2018 Accepted: 5 February 2019

Published online: 21 February 2019

\section{References}

1. Kelly IP, Easley ME. Treatment of stage 3 adult acquired flatfoot. Foot Ankle Clin. 2001;6:153-66.

2. Haddad SL, Myerson MS, Younger A, Anderson RB, Davis WH, Manoli A 2nd. Symposium: adult acquired flatfoot deformity. Foot Ankle Int. 2011;32:95-111.

3. Johnson KA, Strom DE. Tibialis posterior tendon dysfunction. Clin Orthop Relat Res. 1989;239:196-206.

4. Myerson MS. Adult acquired flatfoot deformity: treatment of dysfunction of the posterior tibial tendon. Instr Course Lect. 1997;46:393-405.

5. Hiller L, Pinney SJ. Surgical treatment of acquired flatfoot deformity: what is the state of practice among academic foot and ankle surgeons in 2002? Foot Ankle Int. 2003;24:701-5.

6. Zaw H, Calder JD. Operative management options for symptomatic flexible adult acquired flatfoot deformity: a review. Knee Surg Sports Traumatol Arthrosc. 2010;18:135-42.

7. Moher D, Liberati A, Tetzlaff J, Altman DG, Group P. Preferred reporting items for systematic reviews and meta-analyses: the PRISMA statement. PLoS Med. 2009;6:e1000097.

8. Jadad AR, Moore RA, Carroll D, Jenkinson C, Reynolds DJ, Gavaghan DJ, et al. Assessing the quality of reports of randomized clinical trials: is blinding necessary? Control Clin Trials. 1996;17:1-12.
9. Bucher HC, Guyatt GH, Griffith LE, Walter SD. The results of direct and indirect treatment comparisons in meta-analysis of randomized controlled trials. J Clin Epidemiol. 1997;50:683-91.

10. White IR, Barrett JK, Jackson D, Higgins JP. Consistency and inconsistency in network meta-analysis: model estimation using multivariate metaregression. Res Synth Methods. 2012;3:111-25.

11. Higgins JP, Thompson SG, Deeks JJ, Altman DG. Measuring inconsistency in meta-analyses. BMJ. 2003;327:557-60.

12. Riley RD, Higgins JP, Deeks JJ. Interpretation of random effects metaanalyses. BMJ. 2011;342:d549.

13. Chaimani A, Higgins JP, Mavridis D, Spyridonos P, Salanti G. Graphical tools for network meta-analysis in STATA. PLoS One. 2013;8:e76654.

14. Trinquart L, Chatellier G, Ravaud P. Adjustment for reporting bias in network meta-analysis of antidepressant trials. BMC Med Res Methodol. 2012;12:150.

15. Myerson MS, Corrigan J, Thompson F, Schon LC. Tendon transfer combined with calcaneal osteotomy for treatment of posterior tibial tendon insufficiency: a radiological investigation. Foot Ankle Int. 1995;16:712-8.

16. Hintermann B, Valderrabano V, Kundert HP. Lengthening of the lateral column and reconstruction of the medial soft tissue for treatment of acquired flatfoot deformity associated with insufficiency of the posterior tibial tendon. Foot Ankle Int. 1999:20:622-9.

17. Lombardi CM, Dennis LN, Connolly FG, Silhanek AD. Talonavicular joint arthrodesis and Evans calcaneal osteotomy for treatment of posterior tibial tendon dysfunction. J Foot Ankle Surg. 1999;38:116-22.

18. Catanzariti AR, Lee MS, Mendicino RW. Posterior calcaneal displacement osteotomy for adult acquired flatfoot. J Foot Ankle Surg. 2000;39:2-14

19. Sammarco GJ, Hockenbury RT. Treatment of stage II posterior tibial tendon dysfunction with flexor hallucis longus transfer and medial displacement calcaneal osteotomy. Foot Ankle Int. 2001;22:305-12.

20. Guyton GP, Jeng C, Krieger LE, Mann RA. Flexor digitorum longus transfer and medial displacement calcaneal osteotomy for posterior tibial tendon dysfunction: a middle-term clinical follow-up. Foot Ankle Int. 2001;22:627-32.

21. Fayazi AH, Nguyen HV, Juliano PJ. Intermediate term follow-up of calcaneal osteotomy and flexor digitorum longus transfer for treatment of posterior tibial tendon dysfunction. Foot Ankle Int. 2002;23:1107-11.

22. Haeseker GA, Faber FWM. Calcaneo-cuboid distraction arthrodesis combined with medial soft tissue reconstruction for posterior tibial tendon dysfunction stage II. Foot Ankle Surg. 2003;9:157-60.

23. van der Krans A, Louwerens JW, Anderson P. Adult acquired flexible flatfoot, treated by calcaneocuboid distraction arthrodesis, posterior tibial tendon augmentation, and percutaneous Achilles tendon lengthening: a prospective outcome study of 20 patients. Acta Orthop. 2006;77:156-63.

24. Bolt PM, Coy S, Toolan BC. A comparison of lateral column lengthening and medial translational osteotomy of the calcaneus for the reconstruction of adult acquired flatfoot. Foot Ankle Int. 2007;28:1115-23.

25. Tang KL, Zhou JB, Yang HF, Tan XK, Xie MM, Tao X, et al. Triple arthrodesis with osteotomy for the treatment of stage IIB and stage III adult-acquired flatfoot deformity. Zhonghua Yi Xue Za Zhi. 2010;90:2313-6.

26. Philippot R, Wegrzyn J, Besse JL. Arthrodesis of the subtalar and talonavicular joints through a medial surgical approach: a series of 15 cases. Arch Orthop Trauma Surg. 2010;130:599-603.

27. Haeseker GA, Mureau MA, Faber FW. Lateral column lengthening for acquired adult flatfoot deformity caused by posterior tibial tendon dysfunction stage II: a retrospective comparison of calcaneus osteotomy with calcaneocuboid distraction arthrodesis. J Foot Ankle Surg. 2010;49:380-4

28. Basioni Y, El-Ganainy AR, El-Hawary A. Double calcaneal osteotomy and percutaneous tenoplasty for adequate arch restoration in adult flexible flat foot. Int Orthop. 2011;35:47-51.

29. Jordan TH, Rush SM, Hamilton GA, Ford LA. Radiographic outcomes of adult acquired flatfoot corrected by medial column arthrodesis with or without a medializing calcaneal osteotomy. J Foot Ankle Surg. 2011;50:176-81.

30. Cao H, Tang K, Deng Y, Tan X, Zhou B, Tao X, et al. Excision of accessory navicular with reconstruction of posterior tibial tendon insertion on navicular for treatment of flatfoot related with accessory navicular. Zhongguo Xiu Fu Chong Jian Wai Ke Za Zhi. 2012;26:686-90.

31. Niki H, Hirano T, Okada H, Beppu M. Outcome of medial displacement calcaneal osteotomy for correction of adult-acquired flatfoot. Foot Ankle Int. 2012;33:940-6.

32. Iossi M, Johnson JE, McCormick JJ, Klein SE. Short-term radiographic analysis of operative correction of adult acquired flatfoot deformity. Foot Ankle Int. 2013;34:781-91. 
33. Mehta SK, Kellum RB, Robertson GH, Moore AR, Wingerter SA, Tarquinio TA. Radiographic correction of stage III posterior tibial tendon dysfunction with a modified triple arthrodesis. Foot Ankle Int. 2013;34:1355-63.

34. Cao HH, Tang KL, Lu WZ, Xu JZ. Medial displacement calcaneal osteotomy with posterior tibial tendon reconstruction for the flexible flatfoot with symptomatic accessory navicular. J Foot Ankle Surg. 2014;53:539-43.

35. Yu G, Yu T, Yang Y, Li B, Zhu H, Chen K, et al. Double arthrodesis through a single medial incision approach for flatfoot. Zhongguo Xiu Fu Chong Jian Wai Ke Za Zhi. 2014;28:1321-4.

Ready to submit your research? Choose BMC and benefit from:

- fast, convenient online submission

- thorough peer review by experienced researchers in your field

- rapid publication on acceptance

- support for research data, including large and complex data types

- gold Open Access which fosters wider collaboration and increased citations

- maximum visibility for your research: over $100 \mathrm{M}$ website views per year

At $\mathrm{BMC}$, research is always in progress.

Learn more biomedcentral.com/submissions 